\title{
Phase IV Study of Sunitinib in Chinese Patients with Imatinib-Resistant or Imatinib-Intolerant Gastrointestinal Stromal Tumors
}

\author{
Lin Shen · Yan Sun · Jian-Ming Xu • Carlos Linn (D) Q Qiao Wang • \\ Li-Qiang Yang · Shu-Kui Qin
}

Received: April 25, 2017 / Published online: September 25, 2017

(C) The Author(s) 2017. This article is an open access publication

\section{ABSTRACT}

Introduction: Sunitinib is approved in China for treatment of gastrointestinal stromal tumors (GIST), after disease progression on, or intolerance to, imatinib. However, available data from prospective clinical trials on its efficacy and

Enhanced content To view enhanced content for this article go to http://www.medengine.com/Redeem/ 6C4CF060434CFC2F.

C. Linn $(\bowtie)$

36 Floor, CITIC Square, 1168 Nanjing West Road, Shanghai, China

e-mail: carlos.linn@pfizer.com

L. Shen

Peking University Cancer Hospital and Institute, Beijing, China

Y. Sun

Cancer Hospital/Institute, Chinese Academy of Medical Sciences and Peking Union Medical

College, Beijing, China

J.-M. Xu

Affiliated Hospital of the Academy of Military

Medical Sciences, Beijing, China

C. Linn · Q. Wang

Pfizer, Shanghai, China

L.-Q. Yang

Pfizer, San Diego, CA, USA

S.-K. Qin

PLA Cancer Center of Nanjing Bayi Hospital, Nanjing, China safety in Chinese patients is limited. Our objective is to determine the efficacy and safety of sunitinib in Chinese patients with imatinib-resistant/intolerant GIST.

Methods: An open-label, single-arm, multicenter, phase IV study was performed in Chinese patients with imatinib-resistant/intolerant GIST. Sunitinib was administered orally in 6-week cycles of 4 weeks on-treatment $(50 \mathrm{mg}$ once daily) and 2 weeks off-treatment. The primary endpoint was progression-free survival (PFS). Tumors were assessed every 6 weeks for the first 24 weeks and every 12 weeks thereafter. Responses were evaluated according to Response Evaluation Criteria in Solid Tumors (RECIST) version 1.0.

Results: A total of 60 patients were enrolled, of whom 59 were treated with sunitinib. All patients were Asian, and mean age was 55.1 years. Median PFS was 46.4 weeks $(95 \%$ CI 33.6-53.1). An objective response (complete or partial) was observed in 11/58 (19\%) patients. Median overall survival was 111.3 weeks $(95 \%$ CI 75.4-167.1), median time to tumor progression was 47.3 weeks (95\% CI 34.1-59.3), and median time to tumor response was 22.6 weeks (95\% CI 10.4-57.3). The most common adverse events included leukopenia, fatigue, hand-foot syndrome, and neutropenia. No new safety concerns were identified.

Conclusions: This study confirms that sunitinib is active and well tolerated in Chinese 
patients with imatinib-resistant/intolerant GIST.

ClinicalTrials.gov identifier: NCT00793871.

Funding: Pfizer Inc, USA.

Keywords: Chinese; Efficacy; Gastrointestinal stromal tumors; Imatinib-resistant/intolerant; Safety; Sunitinib

\section{INTRODUCTION}

Gastrointestinal stromal tumors (GISTs) are the most common mesenchymal tumor of the gastrointestinal tract $[1,2]$. The majority originate in the stomach or small intestine and are characterized by overexpression of the KIT receptor tyrosine kinase (RTK) and by KIT gene mutations that trigger constitutive receptor activation [1, 3, 4]. Less common GIST subsets are associated with gain-of-function mutations in platelet-derived growth factor receptor (PDGFR)- $\alpha$, or express no known RTK mutation $[2,5]$.

Standard treatment for localized GISTs is complete surgical excision; however, recurrence is common, as are liver and peritoneal metastases [6, 7]. For advanced GIST, conventional chemotherapy and radiation therapy are largely ineffective [8]. Imatinib mesylate, an RTK inhibitor targeting KIT, PDGFR- $\alpha$, PDGFR- $\beta$, and BCR-ABL, is considered standard first-line treatment [7] and has markedly improved outcomes in advanced GIST since its introduction over a decade ago. However, $\sim 10 \%$ do not respond to first-line imatinib therapy (primary resistance), and the great majority who do respond eventually develop disease progression, usually due to secondary resistance mutations in KIT or PDGFR- $\alpha[2,9]$. Although imatinib dose escalation from the standard 400 to $800 \mathrm{mg} /$ day can temporarily overcome resistance in some cases $[10,11]$, this approach is not suitable for all patients.

Sunitinib malate is an oral multitargeted RTK inhibitor with activity against KIT, PDGFR- $\alpha$, PDGFR- $\beta$, vascular endothelial growth factor receptors-1-3, fms-like tyrosine kinase-3 receptor, colony-stimulating factor receptor type $1 \mathrm{R}$, and glial cell line-derived neurotrophic factor receptor (rearranged during transfection) [12-16]. Sunitinib has become standard treatment for imatinib-resistant/intolerant GIST after its approval based on the interim analysis of a randomized phase III study, which showed that time to progression (TTP) - the primary endpoint-was significantly longer with sunitinib $(n=207)$ than with placebo $(n=105 ; 27.3$ vs. 6.4 weeks; hazard ratio $0.33, p<0.0001$ ) [17]. Approved for imatinib-resistant/intolerant GIST in the USA and Europe in January and July 2006 , respectively, sunitinib $(50 \mathrm{mg} /$ day in the 4-weeks-on/2-weeks-off schedule) was licensed for this indication by the China Food and Drug Administration (CFDA) in October 2007. However, only 17 of the 361 patients who ultimately participated in the phase III study were Asian [18] [data on file, Pfizer]. Limited data are available, other than a study in Chinese patients with imatinib-resistant/intolerant GIST that compared the label-approved schedule with the $37.5 \mathrm{mg} /$ day continuous dosing schedule [19]. Hence, additional efficacy and safety data were needed in Chinese patients, to fulfil CFDA requirements for license renewal of sunitinib for this indication. Here, we report results of a phase IV post-approval commitment study designed for this purpose. Its primary objective was to assess progression-free survival (PFS) in Chinese GIST patients after disease progression on, or intolerance to, imatinib. Secondary objectives included evaluation of additional efficacy endpoints and safety and tolerability.

\section{METHODS}

\section{Patients}

The study recruited patients $\geq 18$ years with diagnosis of GIST confirmed by histopathology and not treatable by surgery, radiation, or combined modality therapy with curative intent. Patients had to have unidimensionally measurable disease (one or more malignant tumor masses, measurable by computed tomography $[\mathrm{CT}]$ or magnetic resonance imaging [MRI]) and prior imatinib treatment failed (defined by disease progression according to Response Evaluation Criteria in Solid 
Tumors [RECIST] version 1.0 or World Health Organization criteria or by occurrence of significant toxicity that precluded further treatment). Radiographic evidence of progression on imatinib was confirmed by the principal investigator before enrollment. Imatinib intolerance was defined by life-threatening adverse events (AEs; i.e. grade 4 according to the National Cancer Institute's Common Terminology Criteria for Adverse Events [NCI CTCAE], version 3.0), or by grade $2 / 3$ AEs that occurred at a moderate dose (e.g. $400 \mathrm{mg} /$ day) and persisted despite standard precautionary measures. Other inclusion criteria included: Eastern Cooperative Oncology Group performance status (ECOG PS) $0 / 1$; adequate organ function; last imatinib dose administered $\geq 1$ week before enrollment; and resolution of all acute toxic effects of imatinib, surgery, radiotherapy, or cryotherapy to NCI CTCAE grade $\leq 1$. Signed informed consent was obtained for all patients.

Exclusion criteria included: prior chemotherapy, chemoembolization therapy, immunotherapy, or any other investigational cancer agent after the last imatinib dose; surgery, radiotherapy, and/or cryotherapy of imatinib-resistant disease (except palliative radiotherapy if $\geq 1$ measurable non-irradiated lesion remained); major surgery within 4 weeks or radiation therapy within 2 weeks of study entry; grade 3 hemorrhage within 4 weeks; diagnosis of secondary malignancy within 5 years (except for treated basal cell carcinoma, squamous cell skin cancer or cervical cancer); history of brain metastasis, spinal cord compression, carcinomatous meningitis, or CT/MRI evidence of brain/leptomeningeal disease; cardiovascular disease within 12 months or ongoing cardiac dysrhythmias, atrial fibrillation, or prolonged QT interval; uncontrolled thyroid abnormality or hypertension; ongoing anticoagulant treatment (except low-dose warfarin for deep vein thrombosis prophylaxis); treatment with cytochrome P450 (CYP) 3A4 inhibitors or inducers within 7 or 12 days, respectively; HIV-positive status; and pregnancy/ breastfeeding.

\section{Study Design}

This was an open-label, single-arm, phase IV study performed at four centers in China between November 2008 and June 2014 (ClinicalTrials.gov identifier: NCT00793871). Patients received sunitinib in repeated 6-week cycles comprising 4 weeks of sunitinib $50 \mathrm{mg}$ once daily followed by 2 weeks off-treatment (schedule 4/2). The same schedule was used in the phase III trial of sunitinib in imatinib-resistant/intolerant GIST [17]. Sunitinib was supplied in bottles of 28 hard gelatin capsules, each containing $12.5 \mathrm{mg}$ of drug. Patients were required to return all bottles at the end of each cycle so compliance could be assessed by counting remaining capsules. Dose interruptions and reductions to 37.5 or $25 \mathrm{mg}$ once daily were permitted for dose-limiting toxicity. Patients who required $>6$ weeks interruption (including the 2-week off-treatment period) or doses $<25 \mathrm{mg}$ were discontinued from the study. Other discontinuation criteria included disease progression, requirement for different anticancer treatment, and consent withdrawal. After discontinuation and a 28-day follow-up period, patients were followed up to collect information on further antitumor therapy and survival.

Tumor assessments were performed by CT or MRI at screening ( $\leq 21$ days before treatment start), every 6 weeks for the first 24 weeks of the study and thereafter every 12 weeks, whenever disease progression was suspected, and at discontinuation (if not done within the past 6 weeks [first 24 weeks of the study] or 12 weeks [after week 24]). In the event of partial or complete response, confirmatory imaging was performed $\geq 4$ weeks later. If a patient discontinued without evidence of tumor progression, tumor assessments continued until progression or initiation of another anticancer treatment. The study was conducted in accordance with the principles of the Declaration of Helsinki and in compliance with International Conference on Harmonization Good Clinical Practice guidelines. The protocol was approved by the relevant independent ethics committees. 


\section{Study Outcomes}

Determination of anti-tumor efficacy was based on objective tumor assessments classified per RECIST version 1.0. The primary endpoint was PFS, defined as time from first study treatment to documentation of objective tumor progression or death from any cause, whichever occurred first. Objective response rate (ORR) was calculated as the proportion of patients who had a best response of complete or partial response. Other efficacy outcomes were defined as the time from first treatment to the date of: (1) death due to any cause (overall survival [OS]); (2) first documentation of objective tumor progression or death due to tumor progression (TTP); or (3) first documentation of objective tumor response that was subsequently confirmed (time to tumor response [TTR]). Response duration was defined as the time from first documentation of objective tumor response that was subsequently confirmed to first documentation of objective tumor progression or death due to any cause. Safety outcomes included AEs, clinical laboratory tests, physical examination, ECOG PS, vital signs, and electrocardiograms (ECGs). AE severity was graded according to NCI CTCAE version 3.0.

\section{Statistical Methods}

The planned sample size $(n=60)$ was selected according to CFDA requirements. PFS, OS, TTP, TTR, and response duration were analyzed in the safety population (which included all enrolled patients who started treatment) using the Kaplan-Meier method. Median values for these parameters are presented along with the two-sided 95\% CIs calculated using the Brookmeyer and Crowley method. ORR analysis used the per-protocol population, which included all treated patients with an adequate baseline assessment. The Clopper-Pearson method was used to calculate $95 \%$ CI for ORR.

\section{RESULTS}

\section{Patient Characteristics and Treatment}

Sixty patients were enrolled, 59 of whom were treated with sunitinib and included in the safety population. The per-protocol population excluded the untreated patient plus an additional patient with no reported lesion at baseline. All patients were Asian and approximately two-thirds were male (Table 1 ). Mean age was 55.1 years (range 29-82). The most common locations of primary GIST diagnosis were the stomach and small intestine. Spindle cell histology was the most common histopathology classification. All patients were imatinib-resistant, with the exception of two patients who were imatinib-intolerant.

Median treatment duration was 223 days (range 28-1288), and the median number of treatment cycles commenced and completed were both 8 (range 1-46). Treatment compliance was $\sim 99 \%$. Most patients (49/59 [83.1\%]) received concomitant medications; the most common was granulocyte-colony stimulating factor $(n=28)$.

\section{Efficacy}

\section{Primary Endpoint}

Overall, 47/59 (79.7\%) patients had objective disease progression $(n=41$ [69.5\%]) or died without objective progression $(n=6[10.2 \%])$. Estimated median PFS was 46.4 weeks (95\% CI 33.6-53.1 weeks). Twelve patients were censored for PFS; main reasons were a gap $>24$ weeks between progressive disease or death and the most recent adequate assessment $(n=5)$, receipt of a new anticancer treatment prior to tumor progression $(n=3)$, and entry into follow-up for progression $(n=2)$.

\section{Secondary Endpoints}

The ORR was $11 / 58$ (19.0\% [95\% CI 9.9-31.4]). All 11 patients had a partial response; no complete responses were reported. Thirty-eight 
Table 1 Patient demographics and other baseline characteristics (safety population)

\begin{tabular}{c}
$N=59$ \\
\hline
\end{tabular}

Age, years

$\begin{array}{ll}\text { Mean (SD) } & 55.1(12.2) \\ \text { Range } & 29-82 \\ \text { Male, } n \text { (\%) } & 39(66.1) \\ \text { Asian, } n(\%) & 59(100) \\ \text { BMI, }{ }^{a} \mathrm{~kg} / \mathrm{m}^{2} & \\ \text { Mean (SD) } & 22.7(3.0) \\ \text { Range } & 16.6-28.4 \\ \text { Weight, kg } & \\ \text { Mean (SD) } & 62.6(11.5) \\ \text { Range } & 44.0-84.0 \\ \text { Height, cm } & \\ \text { Mean (SD) } & 165.7(8.2) \\ \text { Range } & 148-184 \\ \text { Location of primary diagnosis, } n(\%) & \\ \text { Stomach } & 24(40.7) \\ \text { Small intestine/bowel } & 18(30.5) \\ \text { Colon } & 2(3.4) \\ \text { Rectum } & 2(3.4) \\ \text { Other } & 13(22.0)\end{array}$

Histopathological classification, $n$ (\%)

Spindle cell

$33(55.9)$

Epithelioid

$2(3.4)$

Mixed spindle cell and epithelioid

Other

Involved disease sites, ${ }^{\mathrm{b}} n(\%)$

\begin{tabular}{ll} 
Liver & $38(64.4)$ \\
Lung & $5(8.5)$ \\
Lymph node: mediastinum & $1(1.7)$ \\
Lymph node distant & $5(8.5)$ \\
Lymph node regional & $1(1.7)$ \\
Pelvis & $26(44.1)$ \\
\hline
\end{tabular}

Table 1 continued

\begin{tabular}{ll}
\hline & $\boldsymbol{N}=\mathbf{5 9}$ \\
\hline Peritoneum & $12(20.3)$ \\
Other & $24(40.7)$ \\
Involved disease sites, ${ }^{\mathrm{b}} \mathrm{n}(\%)$ & \\
1 & $15(25.4)$ \\
2 & $23(39.0)$ \\
3 & $15(25.4)$ \\
4 & $3(5.1)$ \\
$>4$ & $2(3.4)$ \\
Not reported & $1(1.7)$ \\
\hline
\end{tabular}

$B M I$ body mass index, $S D$ standard deviation

${ }^{a}$ Calculated as weight/(height $\left.\times 0.01\right)^{2}$

b Involved sites included both target and non-target sites; sites with multiple lesions were counted once

(65.5\%) patients had stable disease and six (10.3\%) patients had objective progression. No early deaths were reported. By the data cut-off, 50/59 (84.7\%) patients had died: 48 due to GIST, one due to lung infection, and one due to gastrointestinal hemorrhage. Median OS was 111.3 weeks (95\% CI 75.4-167.1 weeks). In total, 9/59 patients (15.3\%) were censored. Of these, seven (11.9\%) patients were alive at the end of study period and two (3.4\%) patients were lost to follow-up. Tumor progression events were seen in $40(67.8 \%)$ patients; median TTP was 47.3 weeks (95\% CI 34.1-59.3 weeks). A total of 19 (32.2\%) patients were censored, with the main reason being off treatment prior to progression $(n=13)$ and death on treatment without progression $(n=3)$. Eleven $(18.6 \%)$ patients showed a confirmed objective tumor response; median TTR was 22.6 weeks (95\% CI 10.4-57.3 weeks). The median response duration was 48.1 weeks (95\% CI 35.0-92.1 weeks).

\section{Safety}

Overall, 58/59 (98.3\%) patients experienced at least one $\mathrm{AE}$; the majority were considered treatment-related. The most common AEs of any grade and causality were leukopenia, 
fatigue, hand-foot syndrome, neutropenia, decreased platelet count, decreased hemoglobin, decreased neutrophil count, increased aspartate aminotransferase, and skin discoloration (Table 2). Grade 4 AEs were reported in eight (13.6\%) patients (neutropenia $[n=3]$ and decreased hemoglobin, anemia, thrombocytopenia, bile duct stenosis, and peripheral motor neuropathy [all $n=1]$ ). Grade 5 AEs were reported in five $(8.5 \%)$ patients (disease progression $[n=3]$ and lung infection and gastrointestinal hemorrhage [both $n=1]$ ). Eleven (18.6\%) patients discontinued due to AEs. Nine (15.3\%) patients had dose reductions due to AEs and $28(47.5 \%)$ temporarily discontinued due to
AEs. Twelve patients experienced serious AEs, seven of which were nonfatal. No deaths and three nonfatal serious AEs (cardiac failure, rectal hemorrhage, and neutropenia) were considered due to study treatment. Clinical laboratory tests, physical examination, ECOG PS, vital signs, and ECGs did not reveal any remarkable findings.

\section{DISCUSSION}

This phase IV post-approval commitment study investigated the efficacy and safety of sunitinib in 59 patients with imatinib-resistant/intolerant

Table 2 Most common adverse events (any causality and any grade; safety population)

\begin{tabular}{|c|c|c|c|c|}
\hline & All grade & Grade 3 & Grade 4 & Grade 5 \\
\hline \multicolumn{5}{|l|}{$n(\%)$} \\
\hline Any adverse event & $58(98.3)$ & $27(45.8)$ & $8(13.6)$ & $5(8.5)^{a}$ \\
\hline Leukopenia & $38(64.4)$ & $8(13.6)$ & 0 & 0 \\
\hline Fatigue & $31(52.5)$ & $3(5.1)$ & 0 & 0 \\
\hline Hand-foot syndrome & $30(50.8)$ & $6(10.2)$ & 0 & 0 \\
\hline Neutropenia & $29(49.2)$ & $8(13.6)$ & $3(5.1)$ & 0 \\
\hline Platelet count decreased & $26(44.1)$ & $10(16.9)$ & 0 & 0 \\
\hline Hemoglobin decreased & $23(39.0)$ & $4(6.8)$ & $1(1.7)$ & 0 \\
\hline Neutrophil count decreased & $22(37.3)$ & $9(15.3)$ & 0 & 0 \\
\hline Aspartate aminotransferase increased & $21(35.6)$ & $2(3.4)$ & 0 & 0 \\
\hline Skin discoloration & $20(33.9)$ & 0 & 0 & 0 \\
\hline White blood cell count decreased & $19(32.2)$ & $3(5.1)$ & 0 & 0 \\
\hline Diarrhea & $18(30.5)$ & $1(1.7)$ & 0 & 0 \\
\hline Hypertension & $17(28.8)$ & $2(3.4)$ & 0 & 0 \\
\hline Proteinuria & $16(27.1)$ & $2(3.4)$ & 0 & 0 \\
\hline Anemia & $15(25.4)$ & $1(1.7)$ & $1(1.7)$ & 0 \\
\hline Thrombocytopenia & $15(25.4)$ & $4(6.8)$ & $1(1.7)$ & 0 \\
\hline Alanine aminotransferase increased & $14(23.7)$ & $2(3.4)$ & 0 & 0 \\
\hline Blood bilirubin increased & $13(22.0)$ & $2(3.4)$ & 0 & 0 \\
\hline Blood urea increased & $13(22.0)$ & 0 & 0 & 0 \\
\hline
\end{tabular}

Data are shown for adverse events that occurred in $\geq 20 \%$ of patients

${ }^{a}$ Lung infection $(n=1 ; 1.7 \%)$, disease progression $(n=3 ; 5.1 \%)$, and gastrointestinal hemorrhage $(n=1 ; 1.7 \%)$ 
GIST at four centers in China. Median PFS was 46.4 weeks $(95 \% \quad$ CI 33.6-53.1). In the placebo-controlled phase III GIST study that provided the basis for approval of sunitinib in China and elsewhere, median PFS at the main interim analysis was 24.1 weeks (95\% CI 11.128.3 weeks) [17]. The ORR in this phase IV study was $19 \%$ and median TTP was 47.3 weeks. In the phase III study, ORR was 7\% and median TTP was 27.3 weeks [17]. Median TTR was 22.6 weeks in this phase IV study and 10.4 weeks in the phase III study [17]. Median OS was 111.3 weeks (95\% CI 75.4-167.1 weeks) in this study and 72.7 weeks [95\% CI 61.383.0 weeks] in the phase III trial [18]. Median OS in this trial (111.3 weeks; i.e. 25.6 months) was similar to that in GIST patients who received sunitinib on an alternate dosing schedule (23.5 months [95\% CI 21.8 27.0 months]) after imatinib failure [20].

As the current study employed a single-arm, nonrandomized controlled design, the patient population enrolled was selective (e.g. patients were relatively young and $64.4 \%$ had only $1-2$ disease sites); hence, comparisons between the phase III study and the current study are limited $[17,18]$. In addition, without imatinib reimbursement in China, imatinib-refractory/intolerant patients in the current study may have switched to sunitinib earlier as part of a clinical trial rather than undergo imatinib dose escalation/salvage. It is possible that the early switching to sunitinib may have contributed to the improved PFS seen in the current study. Furthermore, it is important to note that five patients were censored from the PFS analysis due to a gap $>24$ weeks. This means that five patients in the PFS analysis setting missed at least two tumor assessment time points ( $>24$ weeks), which impacted and limited the interpretation due to censoring of the time point and a much shorter interval being recorded. Finally, favorable results in the current study may have also been due to increased experience with sunitinib (e.g. improved awareness of $\mathrm{AE}$ management). However, the fact that observed efficacy data with sunitinib were at least as favorable as those reported in the phase III study provides reassurance regarding the activity of this multi-targeted RTK inhibitor in Chinese GIST patients for whom imatinib failed or who cannot tolerate imatinib.

As expected, most patients experienced AEs during the study. However, only 19\% discontinued sunitinib due to AEs, and most AEs could be adequately managed by dose reductions and/ or interruptions. In general, the safety profile of sunitinib in Chinese patients in this study was similar to that of the phase III study, in which the most common AEs were hematologic disorders and the most common treatment-related AEs were fatigue, diarrhea, skin discoloration, and nausea [17, 18]. Some differences were noted, such as a higher incidence of hand-foot syndrome in the current study compared with the phase III study (51\% and 13\%, respectively), which are similar to findings from a phase IV study of sunitinib in Chinese patients with metastatic renal cell carcinoma [21]. However, overall, no new safety concerns were identified. These findings are consistent with a population pharmacokinetic analysis performed across 14 studies, which suggested no clinically significant differences in sunitinib pharmacokinetics between Asian and non-Asian patients [22].

Aside from these studies, relatively little is known about the efficacy and safety of sunitinib in Asian GIST patients. However, the available data suggest that sunitinib is active and well tolerated. In a single-arm retrospective study, 55 Chinese patients with recurrent/metastatic GIST, resistant or intolerant to imatinib, received sunitinib for at least one cycle [19]. Median PFS with schedule $4 / 2$ was 30 weeks (95\% CI 12.8-47.2), whereas median PFS with a continuous daily dose of $37.5 \mathrm{mg}$ was 35 weeks (95\% CI 24.6-45.4). Median OS was 86 weeks and most AEs were grade 1/2 [19]. In a single-center retrospective analysis of 88 Korean GIST patients wherein initial imatinib therapy failed, median TTP and OS with sunitinib were 7.1 and 17.6 months, respectively. The most common grade 3/4 AEs included neutropenia, thrombocytopenia, and hand-foot syndrome [23]. A phase I/II study in Japanese patients with imatinib-resistant/intolerant GIST showed that sunitinib pharmacokinetics and clinical outcomes in this population were similar to those in studies of predominantly Western patients [24]. 
Contemporarily, in the personalized medicine era, sunitinib has shown clinical benefit in all major GIST mutational subtypes, particularly in patients with wild-type or KIT exon 9 genotype and against GISTs with secondary KIT exon 13 or 14 mutations. Patients with exon 9 mutations require an escalated dose of imatinib [25], whereas certain patients, especially those with exon 11 mutations, show no benefit from imatinib dose escalation [26]. More recent studies [27, 28] showed improved efficacy in sunitinib-treated patients with primary mutations in KIT exon 9 compared with exon 11. A limitation of the current study is that correlation between mutational status and sunitinib efficacy and safety were not examined. The selection of imatinib-refractory GIST patients based on biomarkers should be explored in Chinese practice.

\section{CONCLUSION}

In conclusion, this study provides confirmation that sunitinib is active and well tolerated in Chinese patients with imatinib-resistant/intolerant GIST. The safety profile of sunitinib was as expected. These data confirm the place of sunitinib in the routine treatment of this patient population.

\section{ACKNOWLEDGEMENTS}

This study was sponsored, funded and conducted by Pfizer Inc. (New York, NY, USA). Employees of Pfizer were involved in the study design and collection, analysis, and interpretation of data. Medical writing support and article publication costs were funded by Pfizer. Medical writing support was provided by Andy Gannon and Fiona Chaplin at ACUMED $^{\circledR}$ (New York, NY, USA), an Ashfield Company, part of UDG Healthcare plc, with funding from Pfizer Inc. Editorial support was provided by Jaya Vas, $\mathrm{PhD}$, of Engage Scientific Solutions, and funded by Pfizer Inc. All named authors meet the International Committee of Medical Journal Editors (ICMJE) criteria for authorship for this manuscript, take responsibility for the integrity of the work as a whole, and have given final approval for the version to be published. All authors had full access to all of the data in this study and take complete responsibility for the integrity of the data and accuracy of the data analysis.

Disclosures. Carlos Linn is a full-time employee of Pfizer, and declares stocks from Pfizer. Qiao Wang is a former Pfizer employee and declares no conflict of interest. Li-Qiang Yang is a former Pfizer employee and declares stocks from Pfizer. Lin Shenn, Yan Sun, Jian-Ming Xu and Shu-Kui Qin have nothing to disclose.

Compliance with Ethics Guidelines. All procedures followed were in accordance with the ethical standards of the responsible committee on human experimentation (institutional and national) and with the Helsinki Declaration of 1964, as revised in 2013. Informed consent was obtained from all patients included in the study. The protocol was approved by the relevant independent ethics committees.

Open Access. This article is distributed under the terms of the Creative Commons Attribution-NonCommercial 4.0 International License (http://creativecommons.org/licenses/ by-nc/4.0/), which permits any noncommercial use, distribution, and reproduction in any medium, provided you give appropriate credit to the original author(s) and the source, provide a link to the Creative Commons license, and indicate if changes were made.

\section{REFERENCES}

1. Miettinen M, Lasota J. Gastrointestinal stromal tumors. Gastroenterol Clin N Am. 2013;42:399-415. doi:10.1016/j.gtc.2013.01.001.

2. Corless CL. Gastrointestinal stromal tumors: what do we know now? Mod Pathol. 2014;27(Suppl 1):S1-16. doi:10.1038/modpathol.2013.173.

3. Hirota S, Isozaki $\mathrm{K}$, Moriyama $\mathrm{Y}$, Hashimoto $\mathrm{K}$, Nishida T, Ishiguro S, Kawano K, Hanada M, Kurata 
A, Takeda M, Muhammad Tunio G, Matsuzawa Y, Kanakura Y, Shinomura Y, Kitamura Y. Gain-of-function mutations of c-kit in human gastrointestinal stromal tumors. Science. 1998;279:577-80. doi:10.1126/science.279.5350. 577.

4. Hirota S, Isozaki K, Nishida T, Kitamura Y. Effects of loss-of-function and gain-of-function mutations of c-kit on the gastrointestinal tract. J Gastroenterol. 2000;35(Suppl 12):75-9.

5. Heinrich MC, Corless CL, Duensing A, McGreevey L, Chen CJ, Joseph N, Singer S, Griffith DJ, Haley A, Town A, Demetri GD, Fletcher CD, Fletcher JA. PDGFRA activating mutations in gastrointestinal stromal tumors. Science. 2003;299:708-10. doi:10. 1126/science. 1079666 .

6. DeMatteo RP, Lewis JJ, Leung D, Mudan SS, Woodruff JM, Brennan MF. Two hundred gastrointestinal stromal tumors: recurrence patterns and prognostic factors for survival. Ann Surg. 2000;231:51-8.

7. ESMO/European Sarcoma Network Working Group. Gastrointestinal stromal tumors: ESMO Clinical Practice Guidelines for diagnosis, treatment and follow-up. Ann Oncol. 2012;23(Suppl 7):vii49-55. doi:10.1093/annonc/mds252.

8. Dematteo RP, Heinrich MC, El-Rifai WM, Demetri G. Clinical management of gastrointestinal stromal tumors: before and after STI-571. Hum Pathol. 2002;33:466-77.

9. Antonescu CR, Besmer P, Guo T, Arkun K, Hom G, Koryotowski B, Leversha MA, Jeffrey PD, Desantis D, Singer S, Brennan MF, Maki RG, DeMatteo RP. Acquired resistance to imatinib in gastrointestinal stromal tumor occurs through secondary gene mutation. Clin Cancer Res. 2005;11:4182-90. doi:10.1158/1078-0432.CCR-04-2245.

10. Zalcberg JR, Verweij J, Casali PG, Le Cesne A, Reichardt P, Blay JY, Schlemmer M, Van Glabbeke M, Brown M, Judson IR, EORTC Soft Tissue and Bone Sarcoma Group, the Italian Sarcoma Group, Australasian Gastrointestinal Trials Group. Outcome of patients with advanced gastro-intestinal stromal tumours crossing over to a daily imatinib dose of $800 \mathrm{mg}$ after progression on $400 \mathrm{mg}$. Eur J Cancer. 2005;41:1751-7. doi:10.1016/j.ejca.2005. 04.034 .

11. Blanke CD, Rankin C, Demetri GD, Ryan CW, von Mehren M, Benjamin RS, Raymond AK, Bramwell VH, Baker LH, Maki RG, Tanaka M, Hecht JR, Heinrich MC, Fletcher CD, Crowley JJ, Borden EC. Phase III randomized, intergroup trial assessing imatinib mesylate at two dose levels in patients with unresectable or metastatic gastrointestinal stromal tumors expressing the kit receptor tyrosine kinase: S0033. J Clin Oncol. 2008;26:626-32. doi:10.1200/JCO.2007.13.4452.

12. Abrams TJ, Lee LB, Murray LJ, Pryer NK, Cherrington JM. SU11248 inhibits KIT and platelet-derived growth factor receptor beta in preclinical models of human small cell lung cancer. Mol Cancer Ther. 2003;2:471-8.

13. Mendel DB, Laird AD, Xin X, Louie SG, Christensen JG, Li G, Schreck RE, Abrams TJ, Ngai TJ, Lee LB, Murray LJ, Carver J, Chan E, Moss KG, Haznedar JO, Sukbuntherng J, Blake RA, Sun L, Tang C, Miller T, Shirazian S, McMahon G, Cherrington JM. In vivo antitumor activity of SU11248, a novel tyrosine kinase inhibitor targeting vascular endothelial growth factor and platelet-derived growth factor receptors: determination of a pharmacokinetic/ pharmacodynamic relationship. Clin Cancer Res. 2003;9:327-37.

14. Murray LJ, Abrams TJ, Long KR, Ngai TJ, Olson LM, Hong W, Keast PK, Brassard JA, O'Farrell AM, Cherrington JM, Pryer NK. SU11248 inhibits tumor growth and CSF-1R-dependent osteolysis in an experimental breast cancer bone metastasis model. Clin Exp Metastasis. 2003;20:757-66.

15. Kim DW, Jo YS, Jung HS, Chung HK, Song JH, Park KC, Park SH, Hwang JH, Rha SY, Kweon GR, Lee SJ, Jo KW, Shong M. An orally administered multitarget tyrosine kinase inhibitor, SU11248, is a novel potent inhibitor of thyroid oncogenic RET/papillary thyroid cancer kinases. J Clin Endocrinol Metab. 2006;91:4070-6. doi:10.1210/jc.2005-2845.

16. O'Farrell AM, Abrams TJ, Yuen HA, Ngai TJ, Louie SG, Yee KW, Wong LM, Hong W, Lee LB, Town A, Smolich BD, Manning WC, Murray LJ, Heinrich MC, Cherrington JM. SU11248 is a novel FLT3 tyrosine kinase inhibitor with potent activity in vitro and in vivo. Blood. 2003;101:3597-605. doi:10. 1182/blood-2002-07-2307.

17. Demetri GD, van Oosterom AT, Garrett CR, Blackstein ME, Shah MH, Verweij J, McArthur G, Judson IR, Heinrich MC, Morgan JA, Desai J, Fletcher CD, George S, Bello CL, Huang X, Baum CM, Casali PG. Efficacy and safety of sunitinib in patients with advanced gastrointestinal stromal tumour after failure of imatinib: a randomised controlled trial. Lancet. 2006;368:1329-38. doi:10.1016/S01406736(06)69446-4.

18. Demetri GD, Garrett CR, Schoffski P, Shah MH, Verweij J, Leyvraz S, Hurwitz HI, Pousa AL, Le Cesne A, Goldstein D, Paz-Ares L, Blay JY, McArthur GA, Xu QC, Huang X, Harmon CS, Tassell V, Cohen DP, Casali PG. Complete longitudinal analyses of the randomized, placebo-controlled, phase III trial of sunitinib in patients with gastrointestinal stromal 
tumor following imatinib failure. Clin Cancer Res. 2012;18:3170-9. doi:10.1158/1078-0432.CCR-113005 .

19. Li J, Gao J, Hong J, Shen L. Efficacy and safety of sunitinib in Chinese patients with imatinib-resistant or -intolerant gastrointestinal stromal tumors. Future Oncol. 2012;8:617-24. doi:10.2217/fon.12. 29.

20. Reichardt P, Kang YK, Rutkowski P, Schuette J, Rosen LS, Seddon B, Yalcin S, Gelderblom H, Williams CC Jr, Fumagalli E, Biasco G, Hurwitz HI, Kaiser PE, Fly K, Matczak E, Chen L, Lechuga MJ, Demetri GD. Clinical outcomes of patients with advanced gastrointestinal stromal tumors: safety and efficacy in a worldwide treatment-use trial of sunitinib. Cancer. 2015;121:1405-13. doi:10.1002/ cncr. 29220 .

21. Qin S, Jin J, Guo J, Wang J, Zhou F, Huang Y, Ren X, Ye D, Wang Q, Pan S. A phase IV multicenter study of the efficacy and safety of sunitinib as first-line therapy in Chinese patients with metastatic renal cell carcinoma (P851). Presented at the Congress of the European Society for Medical Oncology (ESMO), Vienna, Austria, 28 Sept to 2 Oct 2012.

22. Houk BE, Bello CL, Kang D, Amantea M. A population pharmacokinetic meta-analysis of sunitinib malate (SU11248) and its primary metabolite (SU12662) in healthy volunteers and oncology patients. Clin Cancer Res. 2009;15:2497-506. doi:10.1158/1078-0432.CCR-08-1893.

23. Yoon DH, Ryu MH, Ryoo BY, Beck M, Choi DR, Cho Y, Lee JL, Chang HM, Kim TW, Kang YK. Sunitinib as a second-line therapy for advanced GISTs after failure of imatinib: relationship between efficacy and tumor genotype in Korean patients. Invest New Drugs. 2012;30:819-27. doi:10.1007/s10637-0109593-1.

24. Shirao K, Nishida T, Doi T, Komatsu Y, Muro K, Li Y, Ueda E, Ohtsu A. Phase I/II study of sunitinib malate in Japanese patients with gastrointestinal stromal tumor after failure of prior treatment with imatinib mesylate. Invest New Drugs. 2010;28:866-75. doi:10.1007/s10637-009-9306-9.

25. Heinrich MC, Maki RG, Corless CL, Antonescu CR, Harlow A, Griffith D, Town A, McKinley A, Ou WB, Fletcher JA, Fletcher CD, Huang X, Cohen DP, Baum CM, Demetri GD. Primary and secondary kinase genotypes correlate with the biological and clinical activity of sunitinib in imatinib-resistant gastrointestinal stromal tumor. J Clin Oncol. 2008;26:5352-9. doi:10.1200/JCO.2007.15.7461.

26. Debiec-Rychter M, Sciot R, Le Cesne A, Schlemmer $\mathrm{M}$, Hohenberger $\mathrm{P}$, van Oosterom AT, Blay JY, Leyvraz S, Stul M, Casali PG, Zalcberg J, Verweij J, Van Glabbeke M, Hagemeijer A, Judson I, EORTC Soft Tissue and Bone Sarcoma Group, Italian Sarcoma Group, Australasian Gastro-Intestinal Trials Group. KIT mutations and dose selection for imatinib in patients with advanced gastrointestinal stromal tumours. Eur J Cancer. 2006;42:1093-103. doi:10.1016/j.ejca.2006.01.030.

27. Reichardt P, Demetri GD, Gelderblom H, Rutkowski P, Im SA, Gupta S, Kang YK, Schoffski P, Schuette J, Soulieres D, Blay JY, Goldstein D, Fly K, Huang X, Corsaro M, Lechuga MJ, Martini JF, Heinrich MC. Correlation of KIT and PDGFRA mutational status with clinical benefit in patients with gastrointestinal stromal tumor treated with sunitinib in a worldwide treatment-use trial. BMC Cancer. 2016;16:22. doi:10.1186/s12885-016-2051-5.

28. Rutkowski P, Bylina E, Klimczak A, Switaj T, Falkowski S, Kroc J, Lugowska I, Brzeskwiniewicz M, Melerowicz W, Osuch C, Mierzejewska E, Wasielewski K, Wozniak A, Grzesiakowska U, Nowecki ZI, Siedlecki JA, Limon J. The outcome and predictive factors of sunitinib therapy in advanced gastrointestinal stromal tumors (GIST) after imatinib failure-one institution study. BMC Cancer. 2012;12:107. doi:10.1186/1471-2407-12-107. 